\title{
Analysis of thermodynamic processes under high-voltage condenser welding with inductive dynamic drive
}

\author{
Stanislav Nescoromniy ${ }^{1, *}$, and Evgeny Strizhakov ${ }^{1}$ \\ ${ }^{1}$ Don State Technical University, 344000, 1, Gagarin sq., Rostov-on-Don, Russia
}

\begin{abstract}
When obtaining fixed joints of different thickness from dissimilar materials, there arise difficulties associated with various thermophysical properties, and the likelihood of occurrence of intermetallic phases under chemical interaction. The use of super hard modes of thermodynamic effect during high-voltage condenser welding (HCW) provides localizing heat generation in the contact zone of the weld surfaces, activating the surface layers through converging the surfaces with the displacement of molten metal to the periphery of the contact zone and forming bonding in the solid phase.Recommendations are given for the use of technological methods of high-voltage capacitor welding depending on the combinations of welded alloys. A description of the nature and physical processes occurring at each technological reception is given.It is shown that the energy stored in the capacitor banks is spent on heat and power action. Variation of the mass of the induction - dynamic drive pusher and its inductance enables to adjust the ratio of thermal and mechanical components of the HCW process.The energy dispersion analysis of the experimental compounds obtained from dissimilar alloys confirms the absence of diffusion in high-voltage capacitor welding under super-rigid exposure modes.
\end{abstract}

\section{Introduction}

A share of structures made of dissimilar metals and components of different thicknesses used in mechanical engineering, instrument making, and space technology, is increasing annually. This is due to the unique thermophysical properties of the alloys used (melting point, density, electrical conductivity), as well as the mechanical properties and corrosion resistance [1].

Currently, a wide range of methods have been developed and applied for the production of permanent joints from dissimilar metals, which are not free from shortcomings [2, 3].

As a rule, the connectable components in the structures have the same thickness, which positively affects the conditions of heat removal and the formation of the most favorable structure.

When welding dissimilar non-ferrous metals of different thickness and cross-sectional configurations, difficulties occur; they are associated with the duration of the metal being

* Corresponding author: nescoromniy@mail.ru 
kept at the temperatures of intensive grain growth, which adversely affects the further operational properties of the bonding as a whole [4].

One of the techniques to reduce the duration of heating metals is the use of pulsed energy sources, namely, the discharge of a capacitor bank to weld components.

Under the condenser welding, the accumulated energy is expended only on the thermal impact. The applied system that creates a mechanical force to compress the components before or during the welding process does not always provide creating forces to displace oxide films from the joint zone $[5,6]$.

The equipment and technologies used on the basis of low-voltage capacitors allow the welding process to be performed under hard conditions lasting (1-2000) $10^{-3} \mathrm{~s}$. The accumulated energy is spent only on thermal exposure. The mechanical action is carried out by a separate spring drive which has a greater inertia causing a delay in the force effect versus the thermal one [7]. Permanent bodings are often formed in the liquid phase [8].

The use of hard modes, intermediate inserts between hard-to-weld components under the condenser welding does not solve the problem of reducing the occurrence of brittle intermetallic inclusions in the joint zone and minimizing the development of mass transfer.

\section{Research procedure}

The application of pulsed low-inductance capacitors provides the high-voltage condenser welding with an induction-dynamic drive (HCW with IDD) under super-hard exposure modes with a current duration of $(20-400) 10^{-6} \mathrm{~s}$, amplitude up to $300 \cdot 10^{3} \mathrm{~A}$, pulse pressure up to $5 \cdot 10^{6} \mathrm{~N} / \mathrm{m}^{2}$ for obtaining joints in homogeneous and heterogeneous combinations (copper, aluminum, nickel, etc.) [9].

The IDD consists of a dielectric bandage in which the Archimedes spiral is laid and on whose top a duralumin pusher is installed [10].

The proposed block diagram of the HCW device with IDD is shown in Fig. 1. The process is synchronized through the series electrical coupling of an induction-dynamic drive with weld components [11].

A characteristic property of HCW with IDD is a simultaneous use of accumulated energy in the capacitor banks for thermal and mechanical effects in the connection zone. Thermal energy is released directly in the area of the connectable surfaces, cleaning the surfaces through arcing. Mechanical energy is implemented by an induction-dynamic drive through converging, cleaning by the impact and plastic deformation of the weld surfaces [12].

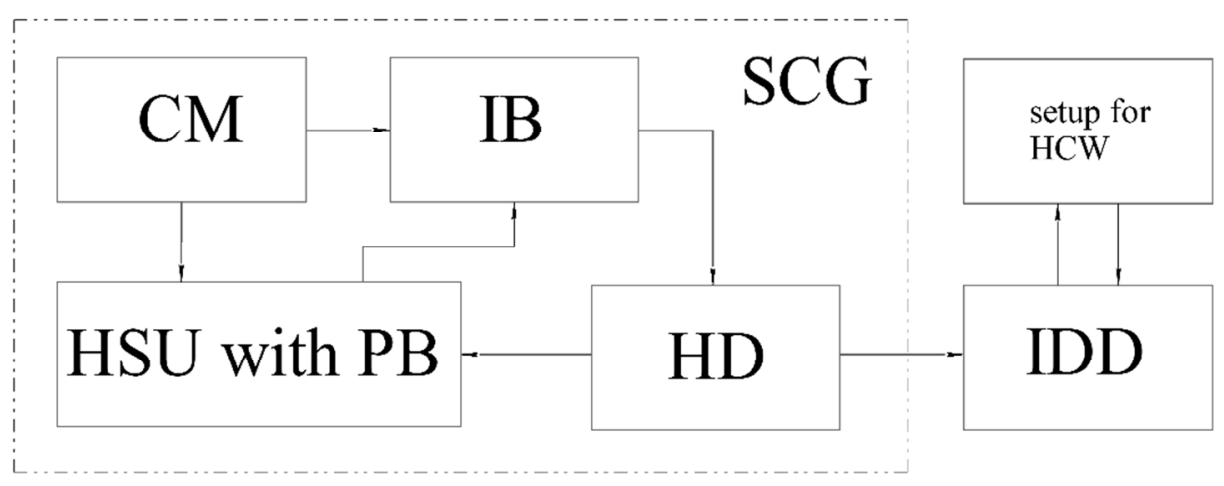

Fig. 1. Block diagram of HCW device with IDD. HSU with PB - high-voltage charging unit with pooling block; HD - high-voltage discharger; CM - control module; IB - ignition block; SCG - sergecurrent generator; IDD - induction-dynamic drive. 
HCW can connect such components as rod-sheet, rod-rod, sheet-sheet, sheet-sphere and of other combinations. But, the most common are rod-sheet bondings.

Depending on the thermophysical and mechanical properties of the weld metals in homogeneous and heterogeneous combinations, the following techniques of $\mathrm{HCW}$ are proposed, Fig. 2 a-c.

It was found that it is preferable to use a protrusion at the end of one of the welded parts to clean the joined surfaces when welding non-ferrous metals with carbon and alloy steels. The protrusion is necessary for closing the electric circuit when a pulse current flows, exciting an electric arc when it explodes, and creating protection of the connection zone with molten metal vapors from the environment, figure 2 a.
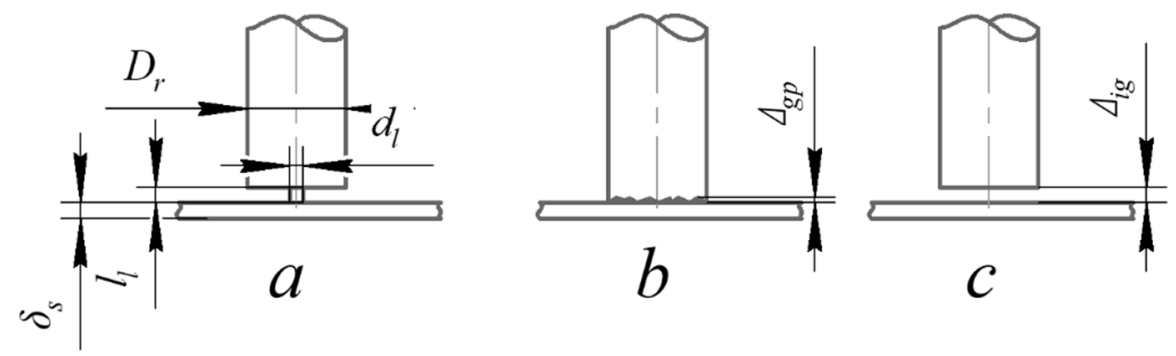

Fig. 2. HCW techniques: $l_{l}$ - length ledge, $d_{l}$ - the diameter of the ledge; $D_{\mathrm{r}}$ - diameter of the rod element $\delta_{\mathrm{s}}$ - the thickness of the sheet element, $\Delta_{\text {g.p. }}$ - length of the gap formed; $\Delta_{i . g .}$ - length of the initial gap.

$\mathrm{HCW}$ with preliminary contact of the welded surfaces, figure $2 \mathrm{~b}$, is recommended for use when connecting non-ferrous metals in a homogeneous combination (aluminum, copper, Nickel alloys).

HCW with a preliminary gap between the parts to be welded is recommended for welding non-ferrous metals in dissimilar combinations, figure $2 \mathrm{c}$.

The HCW parameters behavior for various techniques is shown in figure 3 .

In figure $3 \mathrm{~b}, T_{d}, U, I_{d}$ are similar to figure $3 \mathrm{a}$, they are not indicated; after $\operatorname{arcing}, P_{m}$ and $P_{t}$ match.

When welding without the initial gap and with a protrusion on the end of one of the welded parts, the energy stored in the capacitor banks is spent on the Joule heat generation in the physical contact zone, gorenje electric arc for cleaning the welded surfaces, melting of the ledge, and overcoming the pressure of metal vapors, bringing the surfaces to be connected together, and displacing the molten metal from the connection zone.

In the absence of the initial gap, the thermal effect is limited by the arcing duration, equal to the length of the forming gap as a result of the explosion of microroughness and backlash takeup in the setup. Most of the energy stored in the drive goes to the force impact, in particular, to the deformation of the weld surfaces.

When HCW with the initial gap, the initial gap is broken first, and the electrical circuit is closed. The flowing current pulse heats, vaporizes and melts the welded surfaces, at the same time, due to the magnetic pressure created by the inductor, the pusher brings the welded surfaces closer together. By changing the value of the initial gap $\Delta_{\text {i.g. }}$, the mass of the pusher $M_{p}$ and the inductance of the flat inductor $L$ IDD, you can adjust the duration of the HCW process and change the value of the input thermal and mechanical energy.

The analysis of the HCW process was performed using high-speed photo-recording by the «CORDIN 505» digital electronic camera with an adjustable pulse backlight unit and oscillography - by LeCroy WS424 digital oscilloscope. 


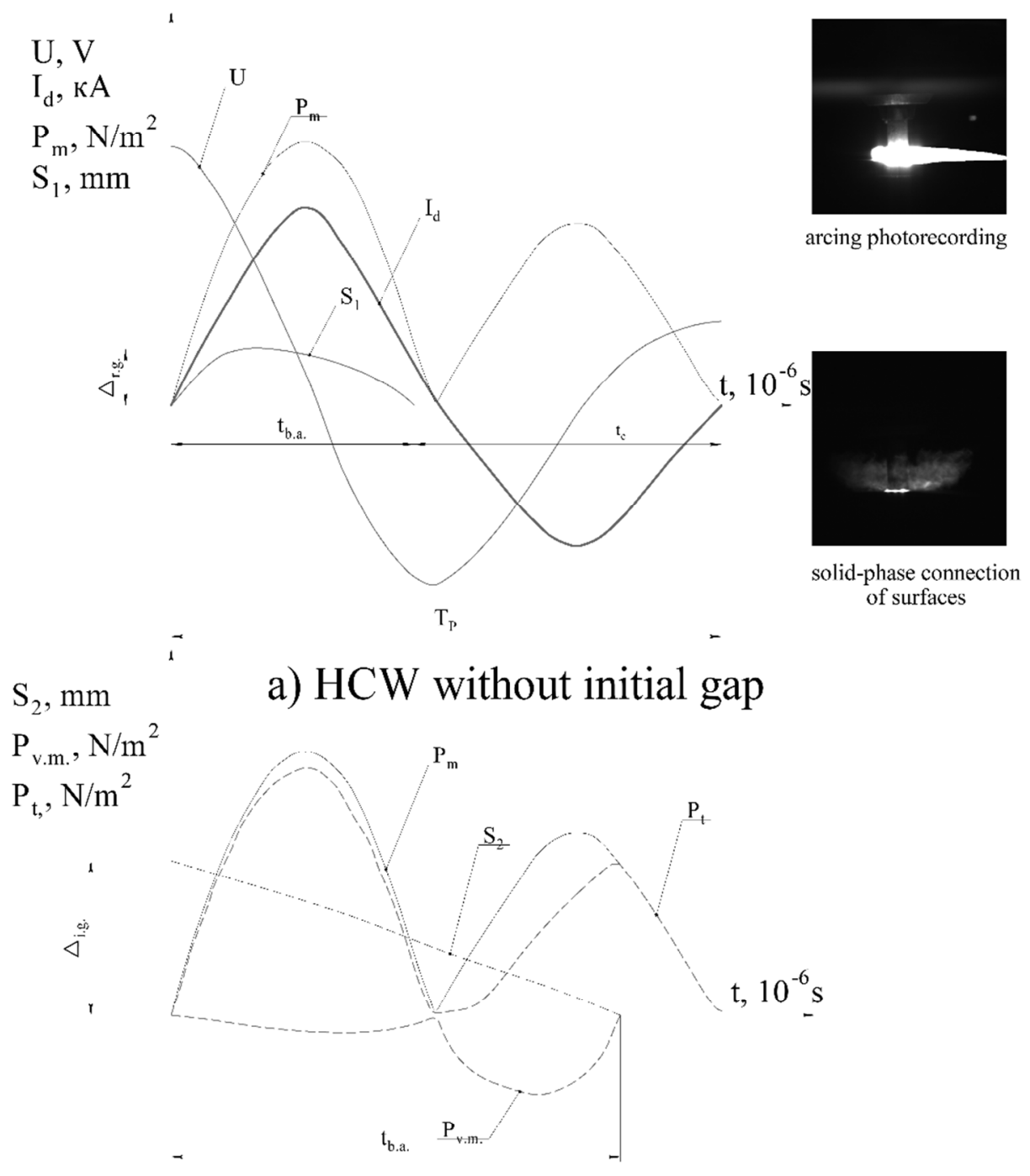

b) HCW with initial gap

Fig. 3. HCW with IDD process behavior. $U$ - voltage on the capacitor; $I_{d}$-discharge current; $T_{d}$ - period of discharge current; $P_{m}$-magnetic pressure; $P_{v m}$ - the vapour pressure of the metal; $P_{t}$ - - total pressure; $\Delta_{r . g}$-the resulting gap; $\Delta_{i . g .}$ - initial gap; $S_{l}$ and $S_{2}$ - moving parts with resulting and initial gaps; $\mathrm{t}_{\text {b.a. }}$ - the burning time of the arc; $\mathrm{t}_{\mathrm{c}}$-solid-phase connection time.

The energy balance under the HCW is described by the expression:

$$
W_{C E S}=W_{h}+W_{m}
$$

where $W_{C E S}$ - is the energy stored in a capacitive energy storage (CES); $W_{h}$ - is the energy spent on heat generation in the connection zone (Joule heat, arcing); $W_{m}$ - is the energy spent on the mechanical impact of the parts to be joined

In expanded form, the equation will be written as follows: 


$$
\frac{C U^{2}}{2}=\left(U I_{d} t_{b . a .}+I_{d}^{2} R t_{c}\right)+\frac{L I_{d}^{2}}{2}
$$

where $C$ - is the capacitance of the capacitive energy storage, F; $U$ - the voltage on the capacitor, $\mathrm{V} ; L$ - inductive reactance of the discharge circuit, determined by the number of turns of the inductor, $\mathrm{H} ; I_{d}$ - discharge current, A; R - is the resistance of the bit setting circuit, $\mathrm{Om} ; t_{c}$-solid-phase connection time $\mathrm{HCW} ; t_{\text {b.a. }}$ - the burning time of the arc

Earlier studies [11] revealed the factors of the HCW process that influence the strength parameters of experimental samples. These include energy parameters of the process: the capacity $\mathrm{C}$ and the charge voltage of the capacitor $U_{c}$, the inductance of the discharge circuit installation HCW $L$ (inductance equipment with induction-dynamic drive) and the geometrical parameters of the assembly ( $\Delta$ i.g. - initial gap, the size of protrusion on the end of the truss element $d_{l}, l_{l}$ ).

The duration of the discharge time of the current $I_{d}$ is determined by the inductance of the discharge circuit of the equipment $L$ with the capacitor block $\mathrm{C}$ and the working inductor. The inductive resistance of the IDD is determined by the number of turns of the inductor $\mathrm{N}$.

\section{Research Results. Discussion}

The most easily implemented indicator of the quality of the obtained compounds is the mechanical strength of the compound when tested for separation $P_{s}$ [13].

The dependence of connection strength from the number of turns of the inductor $\mathrm{N}$ when the charge voltage of the capacitor $\mathrm{U}=2000 \mathrm{~V}$, capacitance of the capacitor unit $\mathrm{C}=2000 \mu \mathrm{F}$, the diameter of the truss element $\mathrm{D}_{\mathrm{r}}=10 \mathrm{~mm}$, the thickness of the sheet $\delta_{\mathrm{s}}=0,8 \mathrm{~mm}$, the diameter and length of the ledge on the end of the truss element $d_{l} / l_{l}=1,3 / 1,5 \mathrm{~mm}$, diameter of inductor $200 \mathrm{~mm}$ alloy B63 is shown in figure 4.

The charge voltage of the capacitor banks $U$ has a significant effect on the strength of the welded joint. If there is a lack of input energy, the HCW process is not implemented, with increasing $U$, the strength of the welded joint increases, but after reaching the extremum, the deformation of the rod element occurs and the strength of the joint decreases.

The capacity of the pooling block $C$ characterizes the energy capabilities of the equipment and, complete with the inductance of the discharge circuit $L$, determines the $\operatorname{intrinsic} f_{i}$ and the working $f_{w}$ frequency of the current pulse [14].

The mass of pusher is $M_{p}$ affects the burning time of the arc $t_{b . a}$ and the amount of plastic deformation $\varepsilon$ and determines the mechanical component of the process.

As a result of the study of experimental samples, the relative local plastic deformation $\varepsilon 1$ was determined depending on the mass of the pusher $M_{p}$, with the diameter of the rod element $D_{r}=10 \mathrm{~mm}$ from the M01 alloy, and the thickness of the sheet $\delta_{\mathrm{s}}=0,8 \mathrm{~mm}$ from the B63, M01 and Amg3 alloys, figure 5.

The decrease in the relative plastic deformation to the left of the extremum is explained by the insufficient value of the mechanical energy $W_{m}$. With an increase in the mass of the pusher to the right of the extremum, there is a delay in the mechanical energy $W_{m}$. with regard to the heat $W_{h}$, an increase in the volume of molten metal in the junction zone, the pressure of metal vapors, which reduces the relative plastic deformation. 


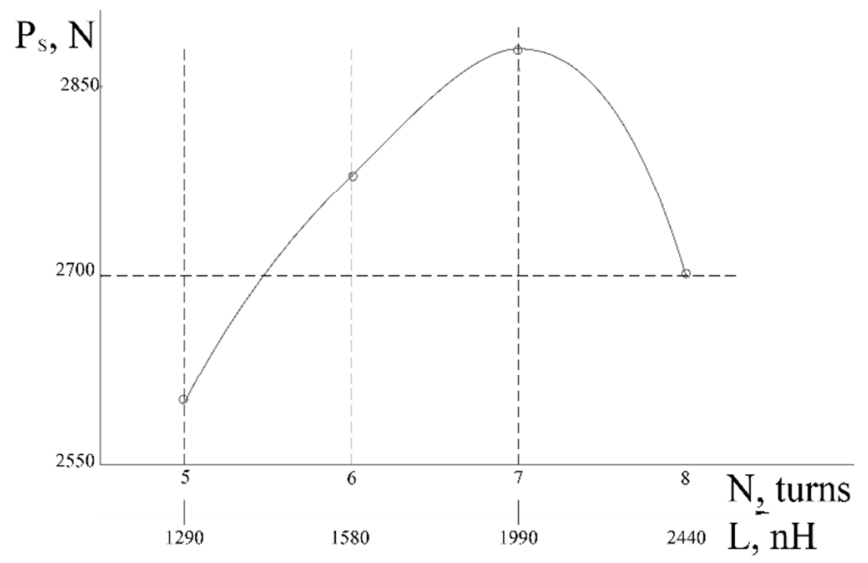

Fig. 4. Dependence of the separation force $\mathrm{P}_{\mathrm{s}}$ on the number of turns of the inductor $\mathrm{N}$ and the inductance corresponding to the number of turns $\mathrm{L}$.

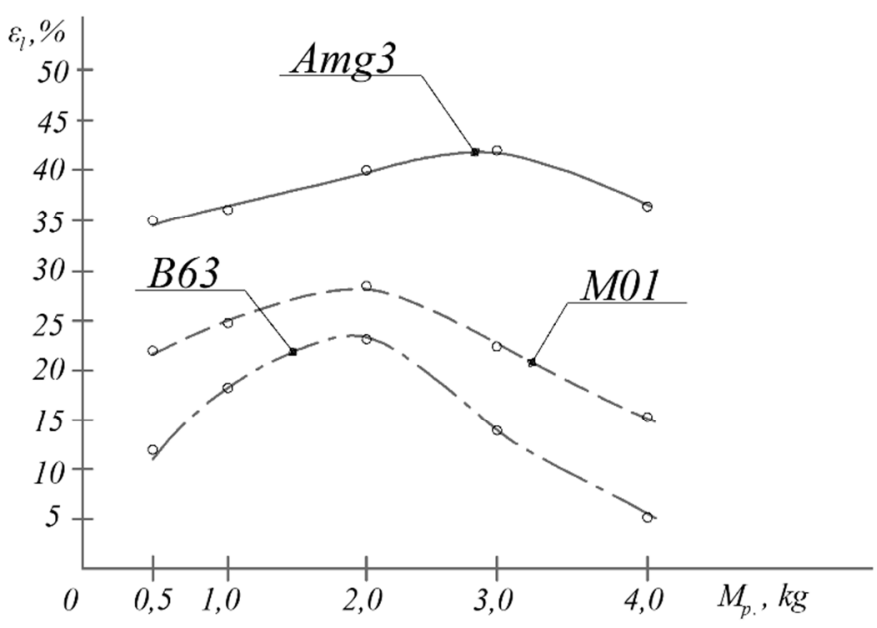

Fig. 5. The dependence of the relative local plastic deformation $\varepsilon_{1}$ on the mass of the pusher $M_{p}$ at $\mathrm{W}$ $=6.5 \mathrm{~kJ}$.

The dependences of the strength of welded joints on the input energy at different weights of the $M_{p}$ pusher are shown in figure 6 .

With an increase in the mass of the follower to the right of $M_{p}$ extremum occurs time lag of mechanical impact $W_{m}$.that does not provide clean mating surfaces due to rapid crystallization of the molten metal.

When the mass of the $M_{p}$ pusher decreases to the left of the extreme, the pulse of specific magnetic pressure created by the pusher is not enough to splash the molten metal out of the zone of connection with oxide films and contaminants [15]. The resulting pressure of metal vapors counteracts the movement of the pusher.

The energy dispersion analysis performed using the Carl Zeiss EVO 50 scanning electron microscope (SEM) of compounds made of dissimilar metals in HCW with IDD is shown in figure 7. 


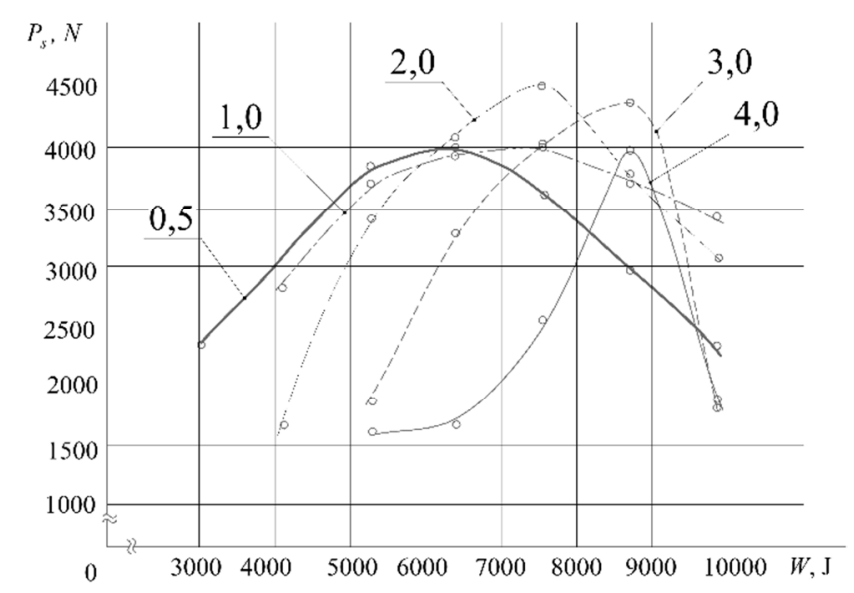

Fig.6. Dependence of the separation force from the input energy $W$ for different pusher mass $M_{p}$ at $\mathrm{N}$ $=7$ turns.

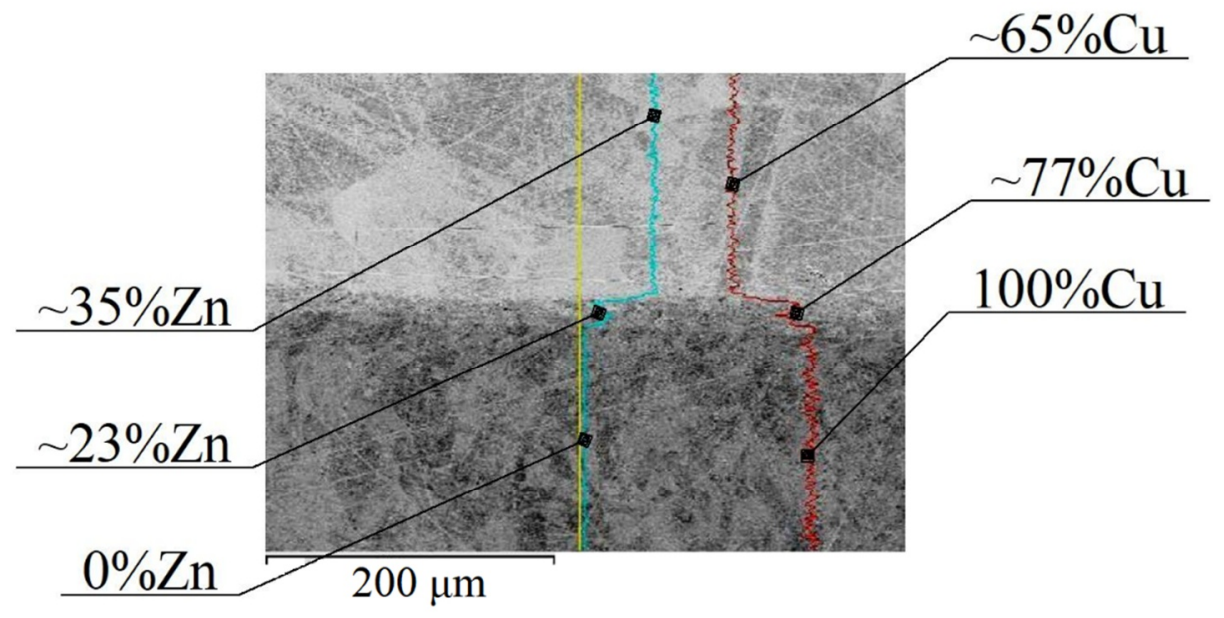

Fig. 7. Elemental composition of bonding zone of alloy B63 and M01 under HCW.

Between the B63 and M01 alloys there is a layer of deformed metal with a width of no more than $20 \mu \mathrm{m}$. The analysis did not reveal the completeness of the diffusion processes, which confirms the assumption about the formation of compounds in the solid phase [16, 17].

\section{Conclusions}

To preserve the original structure and reduce the occurrence of diffusion processes when welding non-ferrous alloys in homogeneous and heterogeneous combinations, it is necessary to apply super-rigid modes of thermal deformation due to the discharge of capacitor banks on the welded parts.

The duration of the thermal and mechanical impact processes can be regulated by the energy parameters of the process, as well as by the parameters of the induction-dynamic drive (pusher weight and inductance). 


\section{References}

1. R. W. Jr. Messler, Joining of Materials and Structures, 285-348 (2004). https://doi.org/10.1016/B978-075067757-8/50006-3

2. P. Groche, M. Becker, C. Pabst, Materials \& Design 118(15), 286-293 (2017). https://doi.org/10.1016/j.matdes.2017.01.013

3. A. G. Anisimov, V. I. Mali, Magnetic pulse welding of different metal sheets 16(1), 151-155 (2019). https://doi.org/10.1016/j.matpr.2019.05.240

4. C. Xiaobing, Z. Zhaoyao, L. Jiehan, Z. Chunhua, Z. Chunya, Journal of Materials $\begin{array}{llll}\text { Processing Technology 2019). } & \text { 204 }\end{array}$ doi.org/10.1016/j.jmatprotec.2018.09.038Get rights and content

5. A. Sezgin, Ş. Mehmet, Journal of Magnetism and Magnetic Materials 502, 15 (2020). https://doi.org/10.1016/j.jmmm.2020.166453

6. E. L. Strizhakov, S.V. Neskoromny, S. O. Ageev, Welding International 29(12), 988 990 (2015). DOI 10.1080/09507116.2015.1012387

7. H. Yohei, S. Yutaro, K. Shinji, Journal of Manufacturing Processes 23, 75-82 (2016). https://doi.org/10.1016/j.jmapro.2016.05.009

8. T. Sapanathan, R. N. Raoelison, N. Buiron, M. Rachik, Joining Technologies, $243-$ 273 (2016). http://dx.doi.org/10.5772/63525

9. E. L. Strizhakov, S.V. Neskoromny, S. O. Ageev, S. V. Lemeshev, Welding International 30 (10), 813 - 816 (2016). DOI 10.1080/09507116.2016.1148409

10. L. Pedro, L. C. Carmen, S. Edmundoda, A. Marco, H. Paulo, F. Juliano, Microelectronics Journal 78, 46-53 (2018). https://doi.org/10.1016/j.mejo.2018.05.012

11. S. V. Nescoromniy, S. O. Ageev, E. L Strizhakov, Key Engineering Materials, 684, 185-192 (2016). DOI: 10.4028/www.scientific.net/KEM.684.185

12. D. Salikhyanov, Contact mechanism between dissimilar materials under plastic deformation. Comptes Rendus Mécanique, 347, 588-600 (2019). https://doi.org/10.1016/j.crme.2019.07.002

13. S.V. Nescoromniy, E. L. Strizhakov, D.V. Rogozin, Procedia Engineering, 150, 930935 (2016). https://doi.org/10.1016/j.proeng.2016.07.065

14. Z. Guoyu , Z. Weihao , H. Di, L. Xiaoxuan, W. Shouxu, H. Yan, C. Yuanming, W. Chong, H. Wei, M. Hua , Z. Jinqun, Journal of Magnetism and Magnetic Materials, 489, 1 (2019). https://doi.org/10.1016/j.jmmm.2019.165363

15. R. Procaccini, W. H. Schreiner, M. Vázquez, S. Ceré, Applied Surface Science, 268(1), 171-178 (2013). https://doi.org/10.1016/j.apsusc.2012.12.050

16. A. G. Anisimov, V. I. Mali, Materials Today: Proceedings, 16(1),151-155 (2019). https://doi.org/10.1016/j.matpr.2019.05.240

17. V. Psyk, C. Scheffler, M. Linnemann, L. Dirk, Procedia Engineering, 207, 353-358 (2017). https://doi.org/10.1016/j.proeng.2017.10.787 